\section{Glitazonas e Sindrome Metabólica: Mecanismos de Ação, Fisiopatologia e Indicações Terapêuticas}

\section{RESUMO}

O diabetes mellitus (DM) é considerado um problema de saúde pública em países devido às suas complicações crônicas macro e microvasculares, com grande impacto na morbimortalidade dos pacientes. A doença é o estágio final de uma síndrome crônica e progressiva, cujas anormalidades fisiopatológicas iniciam-se anos antes do diagnóstico clínico da doença. A síndrome metabólica (SM) é conseqüente ao aumento mundial da prevalência de obesidade. O DM é freqüentemente associado com condições clínicas e laboratoriais que fazem parte da SM, como a obesidade, hipertensão arterial, dislipidemia e microalbuminúria, também fatores de risco cardiovascular. Estudos populacionais demonstram aumento na prevalência de todos os fatores que compõem esta síndrome do pré-diabetes ao DM manifesto, resultando em elevada prevalência de doença cardiovascular e morbimortalidade. Estima-se que $>80 \%$ dos pacientes com DM apresentem SM. As glitazonas são agonistas PPAR- $\gamma$ que melhoram a sensibilidade insulínica. Estas drogas induzem à transcrição de genes relacionados ao metabolismo glicídico e lipídico e à expressão de proteínas inflamatórias e endoteliais associadas com o processo aterosclerótico, resultando em melhora da função endotelial. Entretanto, algumas questões relacionadas às glitazonas merecem mais estudos, como a causa de seus efeitos colaterais (ganho de peso, edema e desenvolvimento de insuficiência cardíaca congestiva). (Arq Bras Endocrinol Metab 2006;50/2:271-280)

Descritores: Glitazonas; Resistência insulínica; Síndrome metabólica; DM2 atualização

\author{
Marilia de Brito Gomes
}

Disciplina de Diabetes da Faculdade de Ciências Médicas, UERJ, Rio de Janeiro, RJ.

\begin{abstract}
Glitazones and the Metabolic Syndrome: Mechanism of Action, Pathophysiology and Therapeutic Indications.

Diabetes mellitus (DM) is considered a major public health problem in both developed and developing countries due to its chronic complications, at the macro or microcirculation, with great impact on mortality and morbidity in all patients. The disease is considered the end of a pathophysiologic process involving peripheral and hepatic insulin resistance and reduced insulin secretion that have been started years before the clinical diagnosis. Metabolic syndrome (MS) is a disorder that results from the increasing prevalence of obesity worldwide. DM is frequently associated with clinical and laboratory features of MS, like abdominal obesity, hypertension, dyslipidemia and microalbuminuria that are also risk factors for cardiovascular disease. Populational studies have demonstrated increasing prevalence of all the features of MS from pre-diabetes to clinical DM resulting in a great risk of cardiovascular disease. The prevalence of MS in DM type 2 is estimated to be $>80 \%$. Glitazones are PPAR- $\gamma$ agonists that improve insulin sensitivity. These drugs induce the transcription of genes related to glucose and lipid metabolism, and expression of inflammatory and endothelial proteins associated with atherosclerosis process resulting in an improvement in endothelial function. However several questions need to be clarified
\end{abstract}

Recebido em 30/10/05 Aceito em 17/01/06 
regarding the glitazones, in special those associated with their adverse effects such as weight gain, edema and heart failure. (Arq Bras Endocrinol Metab 2006;50/2:271-280)

Keywords: Glitazones; Insulin resistance; Metabolic syndrome; DM2

$\mathbf{N}$ as últimas décadas, o diabetes mellitus (DM) tem se tornado um sério e crescente problema de saúde pública nos países desenvolvidos e em desenvolvimento, devido ao aumento de sua prevalência, morbidade e mortalidade. Estimativas recentes da Organização Mundial de Saúde (OMS) projetam um aumento significativo do número de indivíduos com DM até $\mathrm{o}$ ano de 2030. Nessa data, espera-se um universo de mais de 300 milhões de indivíduos com DM (1) (figura 1 ). $\mathrm{O}$ dado mais preocupante desta estimativa é que o número de indivíduos com pré-diabetes definido como intolerância à glicose de jejum (IFG) e intolerância à glicose pós-prandial (IGT) será de aproximadamente 500 milhões (2).

Estudo multicêntrico nacional realizado na década de 80 mostrou que a prevalência média de DM na faixa etária de 30 a 70 anos no Brasil era de 7,6\%, com cerca de 30 a $50 \%$ dos casos não diagnosticados (3). Entretanto, dados mais recentes da região de Ribeirão Preto (interior do Estado de São Paulo) demonstraram que essa prevalência pode ter aumentado em aproximadamente cinco pontos porcentuais (4). De acordo com estes dados, pode-se estimar que ainda neste século nosso país terá aproximadamente 11 milhões de indivíduos com DM. Relatos do Sistema Único de Saúde (DATASUS) mostram que o DM é a quinta indicação de hospitalização e está entre as dez maiores causas de mortalidade no país (5).

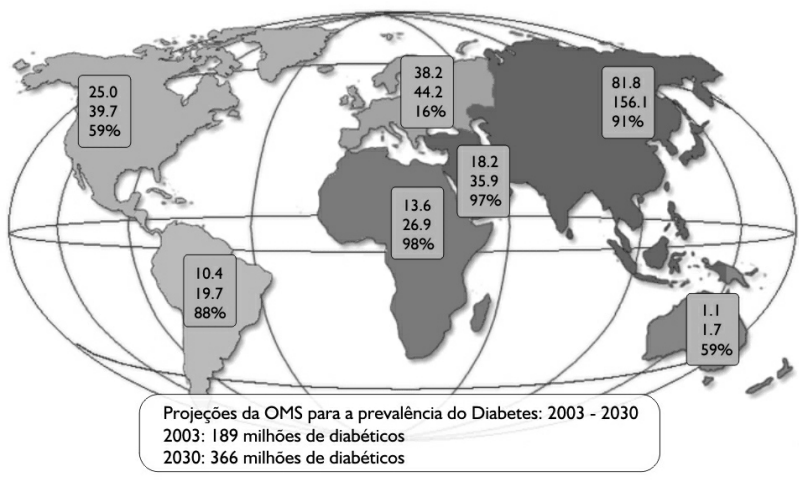

Figura 1. Prevalência mundial do diabetes e projeção de aumento até 2030.
O DM representa um grupo heterogêneo de doenças com múltiplas etiologias, sendo caracterizado por alterações na secreção e ação da insulina, resultando em hiperglicemia crônica com distúrbios no metabolismo dos carboidratos, proteínas e lipídios (6). Considera-se hoje que a doença é o estágio final de uma síndrome crônica e progressiva cujas anormalidades na secreção de insulina pela célula beta e na resistência insulínica muscular, hepática e do tecido adiposo iniciam-se anos antes do diagnóstico clínico da doença (6) e são causadas por componentes genéticos e adquiridos do meio ambiente. Um dos maiores fatores de risco para o desenvolvimento do DM é a obesidade, principalmente a abdominal, a qual também é o principal critério diagnóstico da síndrome metabólica de acordo com a OMS (7). O DM é freqüentemente associado com as outras condições clínicas e laboratoriais que fazem parte desta síndrome, como a hipertensão arterial, dislipidemia, microalbuminúria, que irão exercer um efeito sinérgico no risco cardiovascular dos pacientes (8) (figura 2). Diferentes estudos populacionais têm demonstrado que há aumento da prevalência de todos os fatores que fazem parte desta síndrome do pré-diabetes ao DM manifesto $(9,10)$. A elevada prevalência de doença cardiovascular, além de ser uma importante causa de morbimortalidade (11), é de grande impacto econômico na sociedade, como recentemente destacado em estudo envolvendo os custos da doença nos Estados Unidos em 2002 (12).

Atualmente, temos evidências suficientes de que o melhor controle da glicemia, da pressão arterial e da dislipidemia resulta em uma redução significativa nas complicações microvasculares, no desenvolvimento da doença arterial coronariana e cerebrovascular e mesmo de óbito (13). Com base nesses estudos, foram estabelecidos os níveis ideais de bom controle glicêmico, pressão arterial e lípides, por várias associações diabéticas internacionais e nacionais através de diretrizes. Tem se observado, entretanto, que esses níveis muitas vezes são

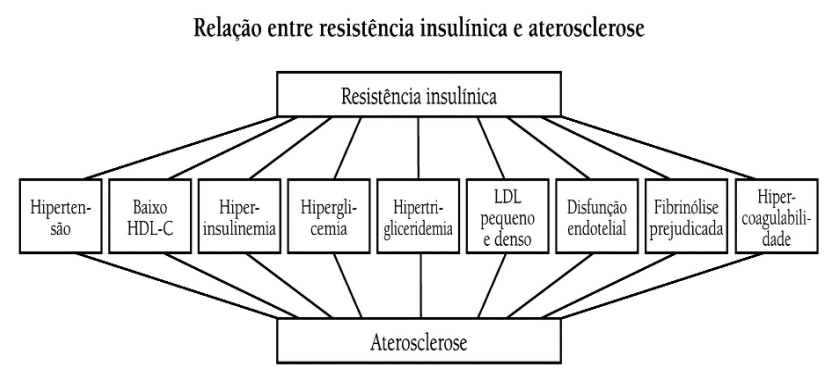

Figura 2. Resistência insulínica e aterosclerose. 
difíceis de serem atingidos na rotina clínica (14). Fatores socioeconômicos, como o elevado custo de tiras reagentes para monitorização da glicemia capilar e fornecimento irregular de medicação (drogas orais e insulina) pelo Sistema Nacional de Saúde, devem colaborar, nesse sentido, em países em desenvolvimento, como o Brasil. Entretanto, é importante enfatizar que como estes pacientes agregam várias comorbidades associadas à resistência insulínica (hipertensão, dislipidemia, distúrbios da coagulação e da fibrinólise), a utilização de diferentes intervenções terapêuticas se faz necessária, dificultando a aderência ao tratamento e, portanto, a obtenção dos alvos clínicos e laboratoriais (15).

Dentro deste contexto, é importante destacarmos as inovações terapêuticas na área de DM que surgiram recentemente no mercado farmacêutico, com mecanismos de ação distintos das drogas previamente existentes porque atuam principalmente nos mecanismos diretos e/ou indiretos que causam a síndrome de resistência insulínica (16).

\section{TIAZOLIDINEDIONAS (GLITAZONAS): ESTRUTURA QUÍMICA}

As tiazolidinedionas, glitazonas, são drogas sensibilizadoras da ação da insulina e representam hoje $21 \%$ das prescrições para pacientes com DM tipo 2 nos EUA e 5\% na Europa (17). Apresentam, como característica de sua estrutura química, um anel diona, que confere a atividade anti-hiperglicêmica que, entretanto, depende da presença da insulina. O restante da molécula difere entre as drogas do grupo e é responsável pela especificidade farmacodinâmica e farmacocinética (18). Atualmente, os fármacos do grupo disponíveis no mercado são a pioglitazona e a rosiglitazona (figura 3 ).

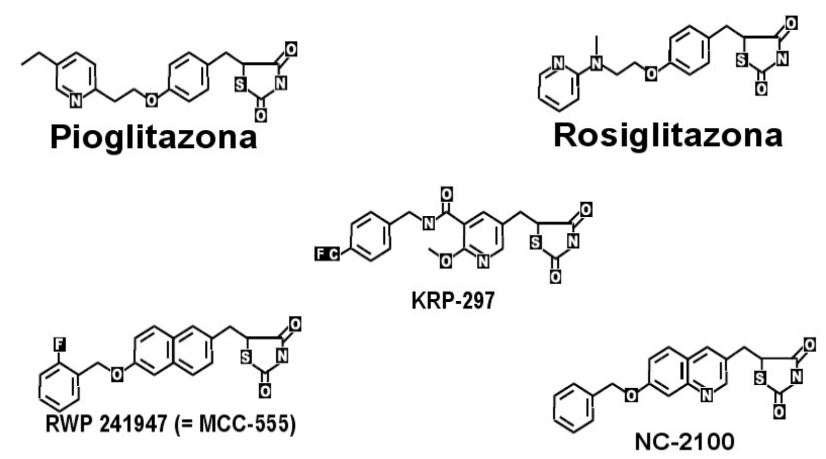

Figura 3. Estrutura química das principais glitazonas e derivados em estudo.

\section{ATIVADORES DOS RECEPTORES PROLIFERADORES DOS PEROXIMAS (PPARS) E OUTROS RECEPTORES NUCLEARES}

As glitazonas têm como mecanismo de ação uma ligação aos receptores nucleares PPARs (peroxisomeproliferator-activated receptors) do tipo $\gamma$. Após a ligação, há uma mudança conformacional no receptor, que permite a ligação com receptor do ácido retinóico (RXR) e recrutamento de um ou mais co-ativadores. A interação deste complexo heterodímero com regiões nucleares responsivas irá determinar a transcrição de aproximadamente 500 genes, sendo que, deste universo, os de nosso conhecimento ainda são minoria, sendo relacionados principalmente ao metabolismo lipídico, glicídico e diferenciação celular (19). Um outro mecanismo de ação do PPAR $\gamma$ independeria de ligação direta com regiões responsivas do DNA e resultaria em repressão à transcrição genética. Este mecanismo de ação estaria associado à atividade antiinflamatória e potencialmente anti-aterogênica do PPAR $\gamma$ através da repressão do fator de transcrição nuclear kapa B (NF-kb). Este fator é responsável pela transcrição de diferentes citoquinas, moléculas de adesão, metaloproteinases e outras proteínas que participam do processo aterosclerótico (20). Um outro possível mecanismo de ação também independente da ligação do agonista com receptor PPAR $\gamma$ foi recentemente descrito e estaria relacionado a uma influência na função mitocondrial (21). Esta ação independente se daria por inibição do complexo 1 mitocondrial, alterando o estado de energia celular.

$\mathrm{O}$ receptor PPARy é expresso principalmente no tecido adiposo, mas também é expresso pela célula beta, endotélio e macrófago (22), e recentemente foi descrito também nas células hematopoiéticas (23). Seu principal ligante endógeno é um derivado da prostaglandina D2 (15 DPGJ $)_{2}$ ) resultante do metabolismo do ácido araquidônico (22). Também são descritos como ligantes, metabólitos derivados da ação da 15lipoxegenase no ácido linoleico (24).

Além do PPAR $\gamma$, outros receptores PPARs foram descritos: $\alpha, \beta$ e $\delta$. O receptor PPAR $\alpha$ é expresso predominantemente no fígado e também pode ser encontrado em músculo, coração, endotélio e nos rins. Tem como principais ligantes endógenos os ácidos graxos livres, e exógenos os fibratos. Os receptores PPAR $\alpha$ são responsáveis pela oxidação de ácidos graxos livres e pela transcrição de fatores com atividade anti-inflamatória e de genes regulatórios das 
lipoproteínas (aumenta a transcrição de apoA I e apoA II e diminui a transcrição de apoC III). As ações nas lipoproteínas resultam em aumento do HDL e maior clearance de triglicerídios exógenos (quilomicrons) e endógenos (VLDL) por maior atividade da lipase lipoprotéica (22). O receptor PPARd é expresso no fígado, músculo, intestino e tecido adiposo, sua ação fisiológica é menos estabelecida e seria mais relacionada ao metabolismo lipídico atuando na oxidação dos ácidos graxos (22). Vários fatores interferem na expressão e ativação de receptores PPARs, dos quais o mais importante é a alimentação. No período de jejum haveria maior atividade de PPAR $\alpha$ em relação a PPAR $\gamma$. No período absortivo, há maior atividade $\operatorname{PPAR} \gamma$.

Os PPARs fazem parte de uma família de receptores nucleares cujos ligantes foram recentemente estabelecidos. Fazem parte desta família o LXR (receptor hepático), cujo ligante são os oxisteróis, e o FXR (receptor farnesoide), cujo ligante são os ácidos biliares. Estes receptores têm uma interação com os PPARs na transcrição genética de genes responsáveis pela regulação do metabolismo lipídico (19).

\section{GLITAZONAS, RESISTÊNCIA INSULÍNICA, ÁCIDOS GRAXOS LIVRES, MODULAÇÃO DO TECIDO ADIPOSO E DOS TRIGLICERIDDEOS E ADIPONECTINAS}

Usualmente, consideramos que as glitazonas melhoram a resistência insulínica porque interferem em diferentes mecanismos no tecido adiposo, músculo e fígado.

O efeito das glitazonas no remodelamento do tecido adiposo resulta em melhora da sensibilidade à insulina, já que interfere na liberação de sinais que agem no tecido muscular e no fígado, dos quais os mais estudados são os ácidos graxos livres (AGL), o fator de necrose tumoral $\alpha(\mathrm{TNF} \alpha)$ e a adiponectina $(25,26)$. As glitazonas diminuem os AGL em cerca de $25 \%$, diminuem a secreção de TNF $\alpha$ e aumentam a concentração sérica de adiponectina, como recentemente observado por diferentes estudos duplo cegos e controlados com placebo e pioglitazona e/ou rosiglitazona em pacientes submetidos ao clamp insulínico euglicêmico (25). Nestes estudos, observou-se diminuição de HbAlc, AGL, TNF $\alpha$ e da produção hepática de glicose. Concomitantemente, houve também aumento da captação de glicose e da concentração sérica de adiponectina. Estes trabalhos permitem a discussão de alguns aspectos fisiopatológicos relacionados à transdução do sinal da insulina. Sabemos que o TNFo induz a fosforilação do primeiro mensageiro da via de transmissão de sinal da insulina, em serina ao invés de tirosina, resultando em dificuldade na transdução do sinal. Além disso, o TNF $\alpha$ induz também um aumento da lipólise e conseqüentemente da liberação de AGL, resultando em lipotoxicidade, o que prejudica a secreção de insulina (27). Níveis diminuídos de adiponectina favorecem a produção hepática de glicose e diminuem a oxidação intra-celular de AGL (muscular e hepático), contribuindo também para o aumento de AGL circulantes. Desta forma, a diminuição de TNF $\alpha$ e o aumento de adiponectina vão resultar em melhora da resistência insulínica muscular e hepática e melhora da secreção de insulina (25). A melhora da resistência insulínica é evidenciada nos diferentes trabalhos pela diminuição do índice HOMA (Homeostasis Model of Assessment) e QUICKI (Quick Insulin Sensivity Index) em pacientes com DM2 (28), como também pela diminuição da insulinemia plasmática (26).

As glitazonas estimulam a diferenciação de adipócitos pequenos, que apresentam como característica maior sensibilidade à insulina e a apoptose dos adipócitos maiores, que são os principais responsáveis pela liberação de ácidos graxos livres, secreção de TNF $\alpha$ e Interleucina 6 (IL-6) (29,30). A diminuição da liberação de AGL tem efeitos benéficos na secreção e na sensibilidade à insulina. As glitazonas vão redirecionar a captação dos AGL para o tecido adiposo porque há estímulo de proteínas como a P2, que se ligam aos AGL e FATP/CD36 que transportam AGL e colesterol para o adipócito (29). No adipócito, o aumento de AGL e a maior atividade da glicerol-quinase também induzida pelas glitazonas (rosiglitazona) resultará em maior formação de triglicerídeos (31). Desta forma, ocorre uma redistribuição do depósito corporal de triglicerídeos, que passa a ser direcionado para o tecido adiposo, com diminuição da gordura visceral e aumento da gordura celular subcutânea (30). De Fronzo e cols., em 2004 (15), demonstraram que, apesar do aumento de peso em pacientes com DM2 em uso de pioglitazona (como monoterapia ou associação com sulfoniluréia) por 16 semanas, houve diminuição significativa da gordura hepática e da produção hepática de glicose, ambos os efeitos associados à diminuição da secreção de resistina (26). 
Os autores sugerem que a pioglitazona possa atuar diminuindo a transcrição do gene da resistina do tecido adiposo visceral. Os diferentes mecanismos de ação das glitazonas no remodelamento do tecido adiposo, inter-relação com as adipocitoquinas, estão descritos na figura 4.

\section{GLITAZONAS, PERFIL LIPÍDICO E CONTROLE GLICÊMICO}

A ativação de PPAR $\gamma$ parece também estimular a transcrição de genes relacionados indiretamente ao metabolismo lipídico e que teriam um efeito benéfico na aterosclerose. Recentemente, foi demonstrado que a rosiglitazona estimularia a expressão do receptor para LDL oxidada (OLRl) em adipócitos, que, normalmente, é expresso em células endoteliais (32). O aumento da expressão do receptor OLRl foi muito maior do que o de CD36, o qual também é um receptor para LDL oxidada. Uma das conclusões deste estudo é que a maior captação de LDL oxidada pelos adipócitos resultaria em diminuição de seus níveis na circulação com possibilidade de inibição da formação de placas ateroscleróticas. Uma outra ação anti-aterogênica das glitazonas (troglitazona e rosiglitazona) foi recentemente relatada na literatura e estaria associada à diminuição da afinidade de ligação entre as partículas de LDL e as moléculas complexas de proteoglicanos do endotélio vascular (33). Esta ação resultaria em diminuição do tempo de permanência da LDL no subendotélio com menor probabilidade de oxidação da partícula e formação das células espumosas. As glitazonas também atuariam no processo de efluxo de colesterol através do estímulo à transcrição do trans-

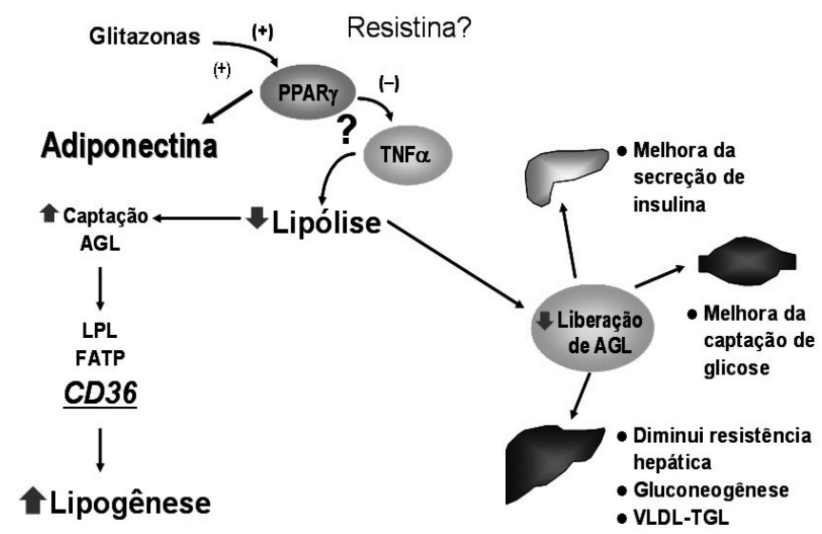

Figura 4. Mecanismos de ação das glitazonas no remodelamento do tecido adiposo e inter-relação com as adipocitoquinas. portador ligador à adenosina trifosfato (ABCAl) (34). Esta proteína, transmembrana, atua no efluxo do colesterol e fosfolipídeos, permitindo que o colesterol se ligue à apolipoproteína A 1 e inicie o processo de transporte reverso (34).

No que se refere aos fatores de risco cardiovasculares clássicos que compõem a síndrome metabólica, recente meta-análise realizada por Chiquette e cols. (35) comparou o efeito da pioglitazona (30 ou $45 \mathrm{mg} / \mathrm{dia}$ ) e rosiglitazona (4 ou $8 \mathrm{mg} / \mathrm{dia}$ ). Nesta meta-análise, foram identificados vinte e três estudos em pacientes com DM2, randomizados e controlados com placebo, que tiveram a duração mínima de 12 semanas e que utilizaram as drogas como monoterapia ou em associações com outras drogas orais ou insulina. No total foram analisados 3.000 pacientes em uso de pioglitazona e 5.000 pacientes em uso de rosiglitazona. De maneira geral, ambas as drogas tiveram efeito semelhante na redução da HbAlc, variando de $(-0,9$ a $-1,26 \%)$, sendo que as maiores reduções foram dosedependentes e relacionadas à associação medicamentosa. O ganho ponderal também foi semelhante e dependente da dose. Foi observada uma heterogeneidade de efeito de ambas as drogas nos parâmetros do metabolismo lipídico. O uso de pioglitazona resultou

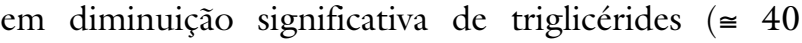

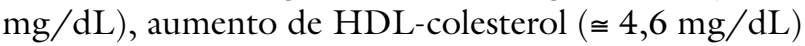
sem ter efeito nos níveis de colesterol total e LDLcolesterol. A rosiglitazona aumentou significativamente o HDL-colesterol $(\cong 2,7 \mathrm{mg} / \mathrm{dL})$, LDL-colesterol

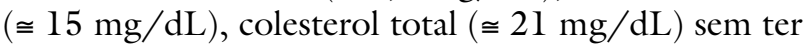
efeito nos níveis de triglicérides e nos níveis de pressão arterial sistólica e diastólica. Nenhum estudo de metaanálise com a pioglitazona avaliou dados de pressão arterial. Nesta meta-análise, o estudo comparativo entre as duas drogas, no que se refere à redução destes fatores de risco cardiovasculares, favoreceu a pioglitazona, sendo que a melhora nos níveis de triglicérides e HDL-colesterol, que fazem parte da síndrome plurimetabólica, foi independente do aumento de peso e da redução da $\mathrm{HbAl}$ c e, portanto, do controle glicêmico. Este efeito foi mais evidente com o uso da pioglitazona, o que poderia ser decorrente de uma maior atividade PPAR $\alpha$ agonista da droga (36).

\section{GLITAZONAS, RESPOSTA INFLAMATÓRIA E FIBRINÓLISE}

As glitazonas, através de sua ação PPAR $\gamma$ agonista, atuariam também em fatores associados à resposta inflamatória, que inter-relacionam a resistência insulí- 
nica com o processo aterosclerótico e doença cardiovascular $(37,38)$. Em alguns trabalhos foi observada diminuição da proteína $C$ reativa ultra-sensível com a pioglitazona (37) e a rosiglitazona (38). Neste último trabalho, Haffner e cols. demonstraram, além da diminuição de proteína $\mathrm{C}$ reativa sérica, diminuição da proteína metaloproteinase 9 responsável pela estabilização da placa aterosclerótica. É discutível se este efeito foi apenas por diminuição de resistência insulínica ou se foi por ação direta da droga.

Além disso, dos mecanismos relacionados à ação

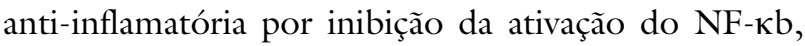
outros também já foram relatados, como a inibição da expressão de moléculas de adesão (ICAM-1), VCAM1, TNFa, metaloproteinase 9 e IL-6 (19). Alguns destes mecanismos também são observados com os agonistas do PPAR $\alpha$ (fibratos) (22) e PPAR (39).

$\mathrm{O}$ aumento dos níveis do inibidor do ativador do plasminogênio (PAI-1) no endotélio é induzido por TNF $\alpha$ e outras citoquinas, sendo um dos fatores que fazem parte do estado pró-trombótico freqüentemente observado no paciente com DM2 e resistência insulínica (8). Estudo in vitro demonstrou que a rosiglitazona e a pioglitazona modularam a expressão de PAI-1 independente da ativação do receptor PPAR $(40)$. A redução dos níveis circulantes de PAI1 em pacientes com DM2 resultaria em melhora do estado pró-trombótico.

\section{GLITAZONAS E FUNÇÃO ENDOTELIAL}

A disfunção endotelial faz parte do processo fisiopatológico da aterosclerose e é caracterizada pela menor produção ou maior inativação do óxido nítrico $(\mathrm{NO})$ (41). A disfunção endotelial é freqüente no paciente diabético em outras condições clínicas que são associadas à síndrome de resistência insulínica (42). Em indivíduos normais, após ligação da insulina com seu receptor há ativação da $\mathrm{NO}$ sintase e, portanto, da produção de NO (43). Na resistência insulínica (DM2, hipertensão e obesidade), este processo encontra-se alterado, sendo no paciente diabético a inativação do NO pelas espécies reativas de oxigênio (ROS) o mecanismo mais provável (44). Os ROS e, portanto, o estresse oxidativo, resultam do efeito da hiperglicemia na ativação de vias metabólicas como a via do poliol, e na ativação de sistemas enzimáticos geradores de superóxido presentes na célula endotelial, como a nicotinamida adenina dinucleotídio fosfato (NADP) oxidase e xantina oxidase (45). Os efeitos lesivos das espécies reativas de oxigênio (ROS) incluem, além da inativação do NO, a lesão do DNA, a ativação da via do diacilglicerol e proteína quinase $\mathrm{C}(\mathrm{DAG} / \mathrm{PKC})$ e o estímulo do fator de transcrição nuclear kapa B (NF-кb). Um dos mecanismos pelo qual as glitazonas melhorariam a função endotelial seria indireto e resultante da diminuição da glicemia e, portanto, da formação de ROS e dos AGL circulantes (41).

Estudos em seres humanos ratificaram estes dados ao demonstrarem que a vasodilatação endotéliodependente avaliada pela reatividade vascular do antebraço com pletismografia melhorou significativamente com uso da rosiglitazona (46), e que outros indicadores precoces da aterosclerose, isto é, a medida da espessura das camadas íntima e média da artéria carótida, diminuíram significativamente com o tratamento com pioglitazona (37).

A rosiglitazona também foi capaz de prevenir a re-estenose em pacientes com DM2 submetidos à implantação de stent (47). Estes trabalhos, observacionais, demonstraram que possivelmente as glitazonas possam exercer um efeito protetor cardiovascular. $\mathrm{Na}$ figura 5 estão demonstradas algumas destas ações protetoras cardiovasculares.

\section{POLIMORFISMO GENÉTICO}

Até o presente momento, quatro mutações genéticas foram descritas no PPAR $\gamma$, sendo a mais comum a Prol2Alanina, que parece estar relacionada com a sensibilidade à insulina. Esta associação foi descrita em pacientes com diabetes tipo 2 , incluindo dados em pacientes brasileiros, e foi associada com menores níveis de insulina e índice HOMA-R (48).

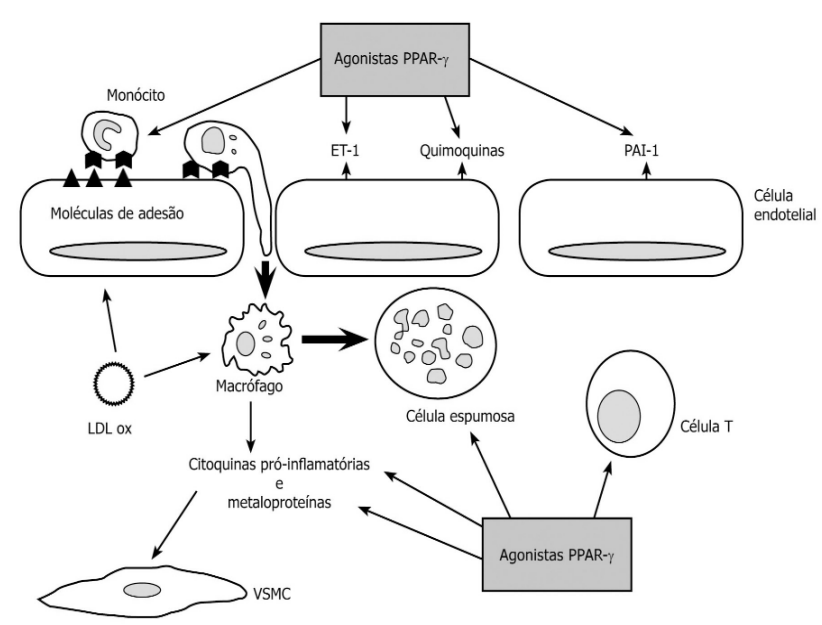

Figura 5. Mecanismos de ação das glitazonas no endotélio. 


\section{EFEITOS COLATERAIS}

\section{Ganho de peso}

É um efeito dose-dependente. O ganho ponderal pode atingir 3,5 a $4 \mathrm{~kg}$ com rosiglitazona na dose de 8 $\mathrm{mg} /$ dia ou pioglitazona na dose de $30-45 \mathrm{mg} /$ dia. A associação de uma das drogas com metformina reduz em $50 \%$ o aumento de peso, principalmente quando a introdução da glitazona é posterior à introdução do metformina (49). Recentemente, DeFronzo descreveu, em um estudo, que o aumento de peso seria preferencialmente por aumento de tecido adiposo e não conseqüente ao edema por aumento do volume plasmático, conforme abaixo descrito (50).

\section{Edema}

Surge em 3 a $5 \%$ dos casos, por aumento do volume plasmático. Apesar de, até o presente momento, não termos a causa estabelecida, as hipóteses mais prováveis são relacionadas ao aumento da reabsorção de sódio e da expressão do fator de crescimento endotelial (51). O edema é mais pronunciado em pacientes que fazem associação de glitazonas com insulina, havendo com mais freqüência o desenvolvimento de insuficiência cardíaca congestiva (ICC), que já é duas a três vezes mais comum em pacientes diabéticos (52). Os fatores de risco mais comuns nas séries estudadas, além daqueles relacionados à insuficiência cardíaca, foram a maior idade e tempo de duração do diabetes. A American Heart Association contra-indica o uso de glitazonas em paciente com insuficiência cardíaca congestiva moderada ou grave (53). Podem surgir problemas em pacientes com insuficiência cardíaca congestiva inicial ou edema periférico já instalado. Este evento fisiopatológico poderia constituir um fator de risco para a ocorrência de edema pulmonar. Recentemente, no estudo de prevenção secundária em pacientes com DM2 com pioglitazona, PROactive (PROspective pioglitAzone Clinical Trial In macroVascular Events), apesar de se ter observado diminuição de $16 \%$ do risco conjunto de morte, enfarte não fatal ou acidente vascular cerebral, a ocorrência de ICC com (6 vs. 4\%) ou sem ( 5 vs. $3 \%$ ) hospitalização foi significativamente maior no grupo que fez uso de pioglitazona (54).

Estes dados foram analisados criticamente pela Prof. Yki-Jarvinen, que concluiu que seria importante termos na prática clínica possíveis preditores de ICC em pacientes em uso de pioglitazona (17). Apesar de não podermos estender estes achados ao uso da rosiglitazona, na Austrália o uso na prática clínica das duas glitazonas foi associado a maior freqüência de ganho de peso, edema periférico e edema pulmonar (52).

\section{Anemia dilucional}

Diminuição do hematócrito pode chegar a 2,5\%, com aparecimento de anemia, necessitando retirada da droga em menos de 1\% dos usuários.

Recentemente, identificou-se a expressão de PPAR $\gamma$ em células hematopoiéticas (23), o que poderia estar associado com os relatos de leucopenia e trombocitopenia nos pacientes diabéticos em uso das glitazonas (55). Enfatizamos, entretanto, que, neste estudo, plaquetas incubadas com rosiglitazona in vitro apresentaram expressão diminuída de mediadores próaterogênicos como tromboxano e CD401.

\section{Hepatotoxicidade}

Apesar de hepatotoxicidade ter sido verificada com o uso de troglitazona, o que motivou retirada da droga do mercado, uma meta-análise realizada por Lebovitz e cols. (56), com 13 estudos em mais de 6.000 pacientes randomizados para uso de rosiglitazona em pesquisas clínicas, mostrou um número muito pequeno de casos com aumento de transaminases acima de 3 vezes do limite superior da normalidade, confirmando que em geral a pioglitazona (57) e a rosiglitazona (56) não induzem a hepatotoxicidade. Recentemente, foi demonstrado que o uso da pioglitazona e a rosiglitazona em pacientes com esteatoepatite não alcoólica resultou em melhora do padrão hepático histológico, diminuição dos níveis de alanina aminotransferase e de gordura hepática (19-58). Os autores atribuem este resultado à melhora da sensibilidade insulínica.

\section{PERSPECTIVAS}

A associação freqüente do DM2 com comorbidades como a dislipidemia tem resultado em investigações sobre associações de agentes terapêuticos que atuem em receptores PPAR $\gamma / \alpha(8)$. Estes agonistas duplos teriam um maior efeito no metabolismo glicídico e lipídico com menos efeitos colaterais (59). Das drogas em estudo, o muriglitazar (60) apresentou efeito semelhante aos agonistas PPAR $\gamma$ no controle glicêmico $\gamma(\downarrow$ HbAlc em 1 a $1,2 \%)$, com aumento do HDL-colesterol em 10 a $16 \%$ e diminuição dos triglicerídeos em 18 a 27\%. Entretanto, apresentaram efeitos colaterais semelhantes aos agonistas PPAR $\gamma$, isto é, edema e ganho de peso, que foram também dose-dependentes. Um outro PPAR $\gamma / \alpha$ agonista, o tesaglitazar, foi utilizado em não diabéticos com características da síndrome metabólica (hipertrigliceridemia e obesidade abdominal) com resultados significativos 
de redução de triglicerídeos, insulina plasmática e ácidos graxos livres e aumento de HDL-colesterol e peso (61). Neste estudo, também foi notada mudança da partícula de LDL-colesterol pequena para de maior diâmetro e, portanto, menos aterogênica.

Outros agentes farmacológicos estão em estudo, como os agonistas para todos receptores PPARs $(\gamma$, $\alpha, \beta$ e $\delta$ ) (39), os agonistas do receptor RXR e os agonistas parciais do receptor $\gamma(62)$, e poderão nos trazer mais possibilidades de intervenções farmacológicas.

Enfatizamos que, apesar da demonstração do efeito na preservação das células beta, com diminuição da incidência cumulativa do diabetes no estudo com troglitazona em mulheres hispânicas com diabetes gestacional (TRIPOD) (63), a terapia farmacológica com as glitazonas em indivíduos com intolerância à glicose ainda não é aprovada pela Sociedade Brasileira de Diabetes (64) e Associação Americana de Diabetes (65).

Os trabalhos acima descritos nos permitem concluir que as glitazonas são indicadas em pacientes com diabetes mellitus tipo 2 que apresentem síndrome de resistência insulínica. Os pontos ainda controversos e merecedores de mais estudos são relacionados aos seus efeitos colaterais, bem como a identificação de mais genes, e de sua função, que são ativados pelos agonistas PPARs.

\section{REFERÊNCIAS}

1. Wild S, Roglic G, Green A, Sicree R, King H. Global prevalences of diabetes. Estimates for the year 2000 and projections for 2030. Diabetes Care 2004; 27:1047-153.

2. http:/www.kenes.com/prediabetes/summary. Acessado em Outubro/2005.

3. Malerbi DA, Franco LJ; The Brazilian Cooperative Group on the Study of Diabetes Prevalence. Multicenter study of the prevalence of diabetes mellitus and impaired glucose tolerance in the urban Brazilian population aged 30-69 yr. Diabetes Care 1992; 15:1509-16.

4. Torquato MTCG, Montenegro RM, Viana LAL, Souza RAGH, Lanna CM, Lucas JCB, et al. Prevalence of diabetes mellitus, impaired glucose tolerance and cardiovascular risk factors in the urban adult population of Ribeirão Preto. Diab Res Clin Pract 2000;50(suppl. 1):S140.

5. DATASUS. http://tabnet.datasus.gov.br/tabnet/tabnet. htm\#Morbidade. Acessado em Setembro/2004

6. Ferrannini E, Gastaldelli A, Miyazaki Y, Matsuda M, Mari A, DeFronzo RA. $\beta$-cell function in subjects spanning the range from normal glucose tolerance to overt diabetes: a new analysis. J Clin Endocrinol Metab 2005:90:493-500.

7. Alberti KGMM, Zimmet P, Shaw J; IDF Epidemiology Task Force Consensus Group. The metabolic syndrome - a new worldwide definition. Lancet 2005;366:1059-62.
8. Eckel RH, Grundy SM, Zimmet PZ. The metabolic syndrome. Lancet 2005;365:1415-28.

9. Isomaa B, Almgren P, Tuomi T, Forsén B, Lahti K, Nissén M, et al. Cardiovascular morbidity and mortality associated with the metabolic syndrome. Diab Care 2001;24(4): 686-9.

10. llane-Parikka $P$, Eriksson JG, Lindström J, Hämäläinen $H$, Kiukaanniemi SK, Laakso M, et al: The Finnish Diabetes Prevention Study Group. Prevalence of the metabolic syndrome and its components. Diabetes Care 2004; 27(9):2135-40.

11. Haffner SM, Lehto S, Ronemaa T, Pyorala K, Laakso M. Mortality from coronary heart disease in subjects with type 2 diabetes and non diabetic subjects with and without prior myocardial infarction. N Engl J Med 1998; 339:229-34

12. American Diabetes Association. Economic costs of diabetes in the US in 2002. Diabetes Care 2003;26:917-32.

13. UK Prospective Diabetes Study Group. Intensive bloodglucose control with sulphonylureas or insulin compared with conventional treatment and risk of complications in patients with type 2 diabetes (UKPDS 33). Lancet 1998:352:837-53.

14. Saydah SH, Fradkin J, Cowie CC. Poor control of risk factors for vascular disease among adults with previously diagnosed diabetes. JAMA 2004;291:335-42.

15. Brown JB, Nichols GA, Perry AP. The burden of treatment failure in type 2 diabetes. Diabetes Care 2004;27: 1535-40.

16. Matthaei S, Stumvoll $M$, Kellerer $M$, Häring HU Pathophysiology and pharmacological treatment of insulin resistance. Endocr Rev 2000;21:585-618.

17. Yki-Jarvinen $\mathrm{H}$. The PROactive study: some answers, many questions. Lancet 2005;366:1240-1.

18. Fürnsinn C, Waldhäusl W. Thiazolidinediones: metabolic actions in vitro. Diabetologia 2002;45:1211-23.

19. Yki-Jarvinen $\mathrm{H}$. Thiazolidinediones. N Engl J Med 2004; 351:1106-18

20. Zhang $X$, Young HA. PPAR and immune system-what do we know. Int Immunopharmacol 2002;2:1029-44.

21. Feinstein DL, Spagnolo A, Akar C, Weinberg G, Murphy P, Gavrilyuk V, et al. Receptor-independent actions of PPAR thiazolidinedione agonists: is mitochondrial function the key? Biochem Pharmacol 2005; 15;70:177-88.

22. WilsonTM, Brown PJ, Sternbach DD, Hence BR. The PPARs: from orphan receptors to drug discovery. J Med Chem 2000;43:527-50.

23. Akbiyik F, Ray DM, Gettings KF, Blumberg N, Francis CW, Phipps RP. Human bone marrow megakaryocytes and platelets express PPAR $y$, and PPARy agonists blunt platelet release of CD40 ligand and thromboxanes. Blood 2004; 104:1361-8.

24. Koshiyama H, Shimono, D, Kuwamura N, Minamikawa, J, Nakamura Y. Inhibitory effect of pioglitzone on carotid arterial wall thickness in type 2 diabetes. J Clin Endocrinol Metab 2001;86(7):3452-60.

25. Martens F, Visseren FLJ, Lemay J, Koning EJP, Rabelink TJ. Metabolic and additional vascular effects of thiazolidinediones. Drugs 2002;62: 1463-80. 
26. Miyazaki Y, Mahankali A, Wajcberg E, Bajaj M, Mandarino LJ, Defronzo RA. Effect of pioglitazone on circulating adipocytokine levels and insulin sensitivity in type 2 diabetic patients. J Clin Endocrinol Metab 2004;89:4312-9.

27. Bajaj M, Suraamornkull S, Hardies LJ, Pratipanawatr T, DeFronzo RA. Plasma resistin concentration, hepatic fat content, and hepatic and peripheral insulin resistance in pioglitazone-treated type II diabetic patients. Int J Obes 2004; 1-7.

28. McGarry JD. Dysregulation of fatty acid metabolism in the etiology of type 2 diabetes. Diabetes 2002;51:7-18.

29. Tan MH, Galtzer B, Johns D, Gilmore KJ. Pioglitazone as monotherapy or in combination with sulfonylurea o metformin enhances insulin sensitivity (HOMA-S or QUICKI) in patients with type 2 diabetes. Curr Med Res Opin 2004;20:723-4.

30. Larsen TM, Toubro S, Astrup A. PPARg agonists in the treatment of type $\|$ diabetes is increased fatness commensurate with long-term efficacy? Int J Obes Relat Metab Disord 2003;27:147-61.

31. Moller DE. New drug targets for type 2 diabetes and the metabolic syndrome. Nature 2001:414:821-7.

32. Guan HP. A futile metabolic cycle activated in adipocytes by antidiabetic agents. Nat Med 2002; 8:1122-8.

33. Chui PC, Guan HP, Leherke M, Lazar MA. PPARg regulates adipocyte cholesterol metabolism via oxidized LDL receptor 1. J Clin Invest 2005; 115:2244-56.

34. Tannock LR, Little PJ, Barret PHR, Wight TN, Chait A. Thiazolidinedionas reduce the LDL binding affinity of non-human primate vascular cell proteoglycans. Diabetologia 2004;47:837-43.

35. Chinetti $G$, Lestavel S, Bocher $V$, Remaley AT, Neve B, Torra IP, et al. PPAR- $\alpha$ and PPAR- $\gamma$ activators induce cholesterol removal from human macrofage foam cells through stimulation of the ABCAl pathway. Nat Med 2001;7(1):53-8

36. Chiquette $E$, Ramirez $G$, DeFronzo R. A meta-analysis comparing the effect of thiazolidinediones on cardiovascular risk factors. Arch Intern Med 2004; 164:2097-14.

37. Henke BR, Blanchard SG, Brackeen MF, Brown KK, Cobb $\mathrm{JE}$, Collins JL, et al. N-(2-Benzoylphenyl)-L-tyrosine PPARgamma agonists. 1. Discovery of a novel series of potent antihyperglycemic and antihyperlipidemic agents. J Med Chem 1998:41:5020-36.

38. Koshiyama $\mathrm{H}$, Shimono D, Kuwamura N, Minamikawa J, Nakamura Y. Inhibitory effect of pioglitzone on carotid arterial wall thickness in type 2 diabetes. J Clin Endocrinol Metab 2001;86(7);3452-60.

39. Haffner SM, Greenberg AS, Weston WM, Chen $H$, Williams K, Freed Ml. Effect of rosiglitazone treatment on non traditional markers of cardiovascular disease in patients with type 2 diabetes mellitus. Circulation 2002; 106:679-84

40. Kurebayashi $S, X u X$, Ishii $S$, Shiraishi $M$, Kouhara $H$, Kasayama S. A novel thiazolidinedione MCC-555 downregulates tumor necrosis factor-alpha-induced expression of vascular cell adhesion molecule-1 in vascular endothelial cells. Atherosclerosis 2005; 182:71-7.
41. Liu HB, Medcalf RL, Simpson RW, Dear AE. Thiazolidinediones inhibit TNF $\alpha$ induction of PAl-1 independent of PPAR $\gamma$ activation. Biochem Biophys Res Commun 2005:334:30-7.

42. Escandon JC, Cipolla M. Diabetes and endothelial dysfunction: a clinical perspective. Endocr Rev 2001; 22:36-52.

43. Dandona P, Aljada A. Endothelial dysfunction in patients with type diabetes and the effects of thiazolidinedione antidiabetic agents. J Diab Complic 2004; 18:91-102.

44. Wang CCL, Goalstone ML, Draznin B. Molecular mechanisms of insulin resistance that impact cardiovascular biology. Diabetes 2005;53:2735-40

45. Brownlee M. Biochemistry and molecular cell biology of diabetic complications. Nature 2001:414:813-20.

46. Schrauwen P, Hesselink MKC. Oxidative capacity, liptoxicity, and mitochondrial damage in type 2 diabetes. Diabetes 2004; $53: 1412-7$.

47. Natali A, Baldweg S, Toschi A, Capaldo B, Barbaro D, Gastaldelli $A$, et al. Vascular effects of improving metabolic control with metformin or rosiglitazone in type 2 diabetes. Diabetes Care 2004;27:1349-57.

48. Choi D, Kim SK, Choi SH, Ko YG, Ahn CW, Jang Y, et al. Preventative effects of rosiglitazone on restenosis after coronary stent implantation in patients with type 2 diabetes. Diabetes Care 2004;27:2654-60.

49. Tavares V, Hirata RD, Rodrigues AC, Monte O, Salles JE, Scalissi $N$, et al. Association between Prol2Ala polymorphism of the PPAR-gamma2 gene and insulin sensitivity in Brazilian patients with type 2 diabetes mellitus. Diabetes Obes Metab 2005;7:605-11.

50. Strowig S, Aviles-Santa ML, Raskin P. Improved glycemic control without weight gain using triple therapy in type 2 diabetes. Diabetes Care 2004:27:1577-83

51. Berria R, Belfort R, DeFillippis E, Cusi K, Mahankali A, Miyasaki $Y$, et al. Fluid retention and hemodilution are not associated with reduction in hematocrit and hemoglobin following pioglitazone and rosiglitazone treatment in type 2 diabetes mellitus. Diabetologia 2005;48(suppl. 1):A17

52. Nesto RW, Bell D, Bonow RO, Fonseca V, Grundy SM, Horton ES, et al. Thiazolidinedione use, fluid retention, and congestive heart failure: a consensus statement from the American Heart Association and American Diabetes Association. Diabetes Care 2004;27:256-63.

53. Husein Z, Wentworth J, Nankervis A, Proietto J, Colman PG. Effectiveness and side effects of thiazolidinediones for type 2 diabetes: real-life experience from a tertiary hospital. MJA 2004; 181:536-9,

54. Nichols GA, Gullion CM, Koro CE, Ephross AS, Brown JB. The incidence of congestive heart failure in type 2 diabetes. Diabetes Care 2004;27:1879-84.

55. Dormandy JA, Charbonnel B, Eckland DJ, Erdmann E, Massi-Benedetti M, Moules IK, et al; The PROactive investigators. Secondary prevention of macrovascular events in patients with type 2 diabetes in the PROactive (PROspective pioglitAzone Clinical Trial In macroVascular Events): a randomized controlled trial. Lancet 2005:366:1279-89. 
56. Digman C, Klein AK, Pittas A. Leukopenia and thrombocitopenia caused by thiazolinediones. Ann Intern Med 2005; 143:465-6.

57. Lebovitz HE, Kreider M, Freed MI. Evaluation of liver function in type 2 diabetic patients during clinical trials. Evidence that rosiglitazone does not cause hepatic dysfunction. Diabetes Care 2002;25:815-21.

58. Schoff C, Lubben $G$. Post-marketing surveillance study of the efficacy and tolerability of pioglitazone in insulin resistant patients with type 2 diabetes in general practice. Clin Drug Invest 2003;23:725-34.

59. Promrat K, Lutchman G, Uwaifo Gl, Freedman RJ, Soza A, Heller T. A pilot study of pioglitazona treatment for nonalcoholic steatohepatitis. Hepatology 2004;39:188-96.

60. Guo Q, Sahoo SP, Wang PR, Milot DP, Ippolito MC, Wu MS, et al. A novel peroxisome proliferator-activated receptor $\alpha / \gamma$ dual agonist demonstrates favorable effects on lipid homeostasis. Endocrinology 2004; $145: 1640-8$

61. Buse JB, Rubin CJ, Friederich R, Viraswami-Appanna K, Lin KC, Montoro R, et al. Muraglitazar, a dual (alpha/gamma) PPAR activator: A randomized, doubleblind, placebo-controlled, 24-week monotherapy trial in adult patients with type 2 diabetes. Clin Ther 2005; 27:1181-95
62. Fargerberg B, Edwards S, Halmos T, Lopatynski J, Schuster H, Stender S, et al. Tesaglitazar, a novel dual peroxima $\gamma / \alpha$ proliferators-activated receptor agonist, dose dependently improves the metabolic abnormalities associated with insulin resistance in a nodiabetic population. Diabetologia 2005;48:1716-25.

63. Pegorier JP. PPAR receptors and insulin sensitivity: new agonists in development. Ann Endocrinol 2005;66:1S10-7.

64. Buchanan TA, Xiang AH, Peters RK, Kjos SL, Marroquin A, Goico J, et al. Preservation of pancreatic $\beta$-cell function and prevention of type 2 diabetes by pharmacological treatment of insulin resistance in high-risk Hispanic women. Diabetes 2002;51:2796-803.

65. http.www.diabetes.org.br/consenso_atual_2002. Acessado em Outubro/2005.

66. American Diabetes Association. Prevention or delay of T2DM. Diabetes Care 2004: 1:S47-S54.

\section{Endereço para correspondência:}

Marília B. Gomes

Disciplina de Diabetes

Av. 28 de Setembro 77, 3ㅇ andar

20551-030 Rio de Janeiro, RJ

Fax: (21) 2587-6324

E-mail: mariliab@uerj.br 